\title{
Práticas integrativas e complementares realizadas em pacientes com lombalgia em uma Unidade Básica de Saúde na região noroeste do Paraná: relato de experiência
}

\author{
Integrative and additional practices carried out on patients with lombalgy in a basic health \\ unit in the northeast region of Paraná: experience report
}
Prácticas integrativas y adicionales realizadas en pacientes con lombalgía en una unidad básica de salud en la región noreste de Paraná: informe de experiência

Bruno Vinicius Resende Mundim ${ }^{1}$, Eduarda Lorenzetti Baldessar', Eduardo Marçal Garcia', Sandro Artur Veit Bublitz', Daniel Luiz Cechinel', Joyce Fernandes da Silva', Isabelle Dieguez Perez de Souza', Vinícius Dino Pozzebon' ${ }^{1}$, Camila Rafaeli Gesualdo', Marcos Benatti Antunes ${ }^{1 *}$.

\section{RESUMO}

Objetivo: Relatar a experiência do uso da auriculoterapia, ventosaterapia, aromoterapia e musicoterapia na redução da dor lombar em pacientes de uma Unidade Básica de Saúde da região noroeste do Paraná. Relato de experiência: $O$ relato foi realizado a partir da vivência de acadêmicos do curso de medicina do Centro Universitária de Maringá em uma Unidade Básica de Saúde do município de Maringá/PR e será apresentado em quatro etapas: a) Disciplina de Interação Comunitária II do curso de Medicina; b) Diagnóstico Local; e c) Planejamento Participativo; e d) Utilização das Práticas Integrativas e Complementares no Sistema Único de Saúde, tendo como público alvo pacientes que frequentam um grupo denominado Grupo de Coluna conduzido pelo Núcleo Ampliado à Saúde da Família. Considerações finais: Com o uso das Práticas Integrativas e Complementares foi possível observar resultados significativos em relação ao relaxamento muscular, tranquilidade, redução do estresse, ansiedade e da dor lombar em pelo menos dois níveis da Escala Visual Analógica da dor de uma sessão a outra, segundo relatos dos pacientes.

Palavras-chave: Terapias complementares, Dor lombar, Atenção primária à saúde.

\section{ABSTRACT}

Objective: To report the experience of the use of auriculotherapy, wind therapy, aromatherapy and music therapy in the reduction of low back pain in patients of a Basic Health Unit in northwestern Paraná. Experience report: The report was based on the experience of medical students from the medical course at the University Center of Maringá in a Basic Health Unit in the city of Maringá / PR and will be presented in four stages: a) Discipline of Community Interaction II of medicine course; b) Local Diagnosis; and c) Participatory Planning; and d) Use of Integrative and Complementary Practices in the Unified Health System, targeting patients who attend a group called the Spine Group conducted by the Extended Family Health Center. Final considerations: With the use of Integrative and Complementary Practices it was possible to observe significant results in relation to muscle relaxation, tranquility, reduction of stress, anxiety and low back pain in at least two levels of the Visual Analogue Scale of pain from one session to another, according to patient reports.

Key words: Complementary therapies, Low back pain, Primary health care.

${ }^{1}$ Centro Universitário de Maringá (UNICESUMAR), Maringá - PR. *E-mail: bena77i@gmail.com 


\section{RESUMEN}

Objetivo: Informar sobre la experiencia del uso de auriculoterapia, terapia de viento, aromaterapia y musicoterapia en la reducción del dolor lumbar en pacientes de una Unidad Básica de Salud en el noroeste de Paraná. Informe de experiencia: El informe se basó en la experiencia de estudiantes de medicina del curso de medicina en el Centro Universitario de Maringá en una Unidad de Salud Básica en la ciudad de Maringá / PR y se presentará en cuatro etapas: a) Disciplina de Interacción Comunitaria II de curso de Medicina; b) diagnóstico local; y c) planificación participativa; y d) Uso de prácticas integrales y complementarias en el Sistema Único de Salud, dirigidas a pacientes que asisten a un grupo llamado Grupo de la columna vertebral dirigido por el Centro de Salud de la Familia Extendida. Consideraciones finales: Con el uso de Prácticas Integrativas y Complementarias, fue posible observar resultados significativos en relación con la relajación muscular, la tranquilidad, la reducción del estrés, la ansiedad y el dolor lumbar en al menos dos niveles de la Escala Visual Analógica del dolor de una sesión a otra, de acuerdo con informes de pacientes.

Palabras clave: Terapias complementarias, Dolor de la región lumbar, Atención primaria de salud.

\section{INTRODUÇÃO}

As Doenças Crônicas Não Transmissíveis (DCNT) constituem-se em um problema de grande importância para o Brasil, que devem ser combatidas, pois, trazem prejuízos sociais, perda da qualidade de vida, limitações as pessoas em suas atividades cotidianas e ao isolamento social (COUTINHO ATQ, et al., 2018). As DCNT são responsáveis por $63 \%$ das mortes no mundo, e no Brasil esse número aumenta para $72 \%$ das causas de morte (WHO, 2011; SCHMIDT MI, et al., 2011).

Além disso, a alta incidência das DCNT leva a um problema econômico, visto que os recursos do Sistema Único de Saúde (SUS) são direcionados ao tratamento dessas doenças acarretando um grande custo para o país (ACHUTTI A e AZAMBUJA MIR, 2004). Além disso, existe também uma união tanto de problemas econômicos quanto sociais, como é o caso da invalidez, que gera uma grande adversidade tanto para 0 indivíduo como para o SUS (SANTOS TR, et al., 2012).

As DCNT ainda têm etiologia múltipla, pois existem muitos fatores envolvidos, podendo ser originada pelas condições e etilo de vida dos indivíduos, incapacidades funcionais, desigualdades sociais e ainda assim, necessitam de uma abordagem holística e sistemática para seu tratamento (WHO, 2005). Nesse contexto, destaca-se a lombalgia, que corresponde a dor na região lombar, podendo causar limitações, redução de funcionalidade, incapacidade e afastamento do trabalho (ABREU ATJB e RIBEIRO CAB, 2010). Sua prevalência é estimada em $70 \%$ a $85 \%$ da população com pelo menos um episódio de dor nas costas durante a vida que podem ser de origem anatômica, fisiológica, osteomuscular ou até mesmo decorrente de uma gestação (SILVA MC, et al., 2004; GOMES MRA, et al., 2013).

Sendo assim, as práticas integrativas e complementares (PIC) são uma forma de tratamento alternativo que podem ser utilizadas como procedimentos para combater e tratar as DCNT. São mais de 29 PIC's oferecidas pelo SUS, dentre elas, destacamos a Medicina Tradicional Chinesa (MTC) com acupuntura e auriculoacupuntura, ventosaterapia, aromoterapia e musicoterapia, que são indicadas isoladas ou coadjuvantes no tratamento de inúmeras patologias, tais como, dependências de drogas lícitas/ilícitas, reabilitação após procedimentos cirúrgicos e acidentes vasculares cerebrais, estresse, ansiedade, cefaleia, epicondilite, fibromialgia, dor miofascial, osteoartrite, lombalgias, asma, entre outras (MINISTÉRIO DA SAÚDE - BRASIL, 2006).

Vale ressaltar que as PIC's foram institucionalizadas no SUS em 2017-2018 e segundo dados oficiais elas estão presentes em até $56 \%$ dos municípios. É uma prática que vem crescendo ao longo dos anos devido a seus resultados positivos. Por volta de $80 \%$ das PIC's ocorrem na atenção primária de saúde (APS), vinculada ao SUS (TESSER CD, et al., 2018). 
Nesse sentido, o objetivo desse estudo foi relatar a experiência do uso da auriculoterapia, ventosaterapia, aromoterapia e musicoterapia na redução da dor lombar em pacientes de uma Unidade Básica de Saúde (UBS) da região noroeste do Paraná.

\section{RELATO DE EXPERIÊNCIA}

O relato de experiência foi realizado a partir da vivência de acadêmicos do curso de medicina do Centro Universitária de Maringá (UNICESUMAR) em uma Unidade Básica de Saúde (UBS) do município de Maringá/PR e será apresentado em quatro etapas: a) Disciplina de Interação Comunitária II do curso de Medicina; b) Diagnóstico Local; e c) Planejamento Participativo; e d) Utilização das Práticas Integrativas e Complementares no (SUS), tendo como público alvo pacientes que frequentam um grupo denominado Grupo de Coluna conduzido pelo Núcleo Ampliado à Saúde da Família (NASF).

\section{Disciplina de Interação Comunitária II do curso de Medicina}

A disciplina de Interação Comunitária (IC) II do curso de Medicina do UNICESUMAR busca ensinar o acadêmico de forma integrada, os conceitos e métodos de vigilância em saúde, por meio da vivência no processo de trabalho e contexto das UBS, tendo como foco as DCNT e a Promoção a Saúde. Associado a isto, a disciplina propõe ao aluno a análise da situação de saúde, planejamentos e realizações de ações e intervenções na comunidade, visando o trabalho de forma cooperativa e integrada em grupo, com postura médica centrada nas necessidades de grupos e comunidades de forma criativa e com compromisso social (CENTRO UNIVERSITÁRIO DE MARINGÁ - UNICESUMAR, 2019).

Nesse contexto, a disciplina de IC II foi essencial no processo de aprendizagem, principalmente no que tange, a forma de acolhimento da comunidade, levando em consideração a diversidade de patologias encontradas. Nesse aspecto, sendo a UBS a principal porta de entrada para o SUS, compreender a sua dinâmica é de suma importância para a formação biopsicossocial do médico que vai além da formação técnica, mas compreende construção de um profissional de saúde que entende o paciente como um ser não exclusivamente biológico, mas, sim, socialmente inserido.

Sendo assim, a experiência de cursar a disciplina de IC II, foi positiva, referindo-se aos conteúdos práticos vivenciado pelos acadêmicos e docente, reforçando a importância de todos estarem inseridos no processo de ensino-aprendizagem, e além disso, oportunizar a vivência em um serviço de saúde com seus protocolos e rotinas de trabalho associados ao processo educacional, assim como da responsabilidade profissional, mesmo sendo considerado um campo de estágio.

\section{Diagnóstico Local}

Com base nas propostas da disciplina de IC II, o grupo realizou o Pentáculo do Bem-Estar de Nahas MV et al. (2000) como forma de classificar o estilo de vida dos pacientes com lombalgia da UBS em Maringá. Este pentáculo engloba os tópicos de alimentação, controle do estresse, relacionamentos sociais, atividade física e comportamento preventivo, composto por um total de 15 questões.

O questionário foi aplicado pelos acadêmicos e contou com a participação de pacientes do Grupo de Coluna local, os resultados mais significativos foram nos componentes de nutrição, atividade física e controle do estresse. Baseado nas informações obtidas com o questionário, o grupo elaborou por meio do planejamento participativo, intervenções para o Grupo de Lombalgia.

\section{Planejamento Participativo}

O planejamento participativo foi o método pelo qual o grupo trabalhou ao longo da disciplina, reunindo-se com a equipe de saúde composta pela direção da unidade, médicos, enfermeiros, técnicos de enfermagem e Agentes Comunitários de Saúde (ACS) para apreciação e discussão das propostas realizadas pelo grupo de IC II a serem realizadas com os pacientes do Grupo de Coluna do NASF.

Na reunião de planejamento participativo, o grupo utilizou um banner em formato de cartaz em formato A3, colorido, para apresentação dos resultados do estilo de vida dos pacientes e a proposta de montar um 
ambulatório de PIC's, proposta essa, que foi bem aceita por toda equipe de saúde. No momento das propostas, a direção disponibilizou dois consultórios médicos para realização das práticas e os ACS sugeriram a divulgação no NASF por meio de cartazes e, além disso se disponibilizaram a convidar os pacientes com lombalgia de suas respectivas micro áreas a participarem do ambulatório.

Esse cenário, proporcionou muito aprendizado tanto aos estudantes e professor, quanto para os profissionais que participaram do planejamento, pois demonstrou parceria entre o Serviço de Saúde e a Instituição de Ensino Superior (IES), pensando juntos no objetivo geral que foi proporcionar qualidade no atendimento e saúde ao paciente.

\section{Utilização das Práticas Integrativas e Complementares no SUS}

Os acadêmicos passaram por capacitação e treinamento prático para realização das PIC's antes de iniciar os atendimentos no ambulatório na UBS. Para o tratamento de lombalgia, utilizamos a auriculoterapia, ventosaterapia, aromoterapia e musicoterapia, as duas últimas apenas para deixar o ambiente mais acolhedor. No consultório 1, o paciente passava por dois momentos do atendimento, primeiro foi aplicado um instrumento de coleta do histórico do paciente contendo a sua história de patologias pregressas e histórico familiar.

Em seguida, realizava-se a anamnese com perguntas direcionada a queixa principal do paciente e o seu estado atual relacionado a dor, aqui aplicava-se a Escala Visual Analógica (EVA) da dor, e ainda aferia os sinais vitais e dados antropométricos do paciente (Figura 1). Essa experiência inicial de observação e aplicação dos questionários foi de grande valia para uma comunicação efetiva, pautada na importância da relação médico-paciente, evidenciou-se de tal forma a conexão e o vínculo entre acadêmicos e pacientes, que muitos exteriorizaram com elogios e carinho o atendimento oferecido na primeira etapa, sem ainda realizarem as PIC's.

Após esses procedimentos, ainda no consultório 1, os acadêmicos realizavam a auriculoterapia com protocolo específico para redução do estresse e ansiedade, utilizando-se da inserção com micropore de cristais ou sementes nos pontos: Shen Men, SNS, Rim, Ansiedade anterior 1 e 2, Ansiedade posterior e Alegria. E para alguns casos de dores intensas na coluna, utilizamos pontos sobre a ante hélice, que é uma cartilagem que está em forma de um "C" envolvendo a Concha, a parte mais interna da orelha.

Durante essa etapa, muitos pacientes questionavam sobre a eficácia do tratamento, quanto tempo deveria ficar com os cristais ou sementes inseridos no pavilhão auricular, se podia lavar a orelha, se sentiria dor, quanto tempo deveria ficar com as sementes ou cristais na orelha, entre outras perguntas. A cada resposta dada pelos acadêmicos, fortalecia os conhecimentos do grupo e dos pacientes, proporcionando segurança e melhores resultados no tratamento.

Já no consultório 2, foi preparado um ambiente acolhedor com aromoterapia (essências relaxantes) e musicoterapia (sons da natureza) para ajudar na meditação e relaxamento dos pacientes. Ao receber o paciente nesse consultório, orientava-se sobre os possíveis hematomas devido a técnica de sucção da ventosaterapia, apresentava-se um termo de consentimento livre e esclarecido sobre esses hematomas e orientava o paciente sobre o benefício desta PIC, sendo esta uma técnica milenar que visa melhorar a circulação sanguínea, aliviar a dor, liberar a tensão em um músculo e dispersar energia acumulada.

Posteriormente a essas orientações, o paciente deitava-se na maca e realizava-se uma massagem nas costas com óleo de uva, em seguida aplicava-se a ventosaterapia nos pontos do meridiano da bexiga (pontos da acupuntura), de acordo com a (Figura 1).

Durante a sessão, os pacientes relaxavam ao ponto de dormir durante o procedimento, além disso, muitos relatavam que foi a primeira experiência com as PIC e que gostariam de continuar com o tratamento, pois já sentiam melhora da dor de imediato, além de tranquilidade e consequentemente redução do estresse e ansiedade. $O$ tratamento foi semanal com duração de quatro semanas. Os pacientes relatavam que a dor minimizava de 1 a 3 níveis, de acordo com a EVA da dor, após a primeira sessão, com queda posterior nos tratamentos das semanas subsequentes. 
Figura 1 - Ambulatório de Práticas Integrativas e Complementares na Unidade Básica de Saúde, MaringáPR, 2019.

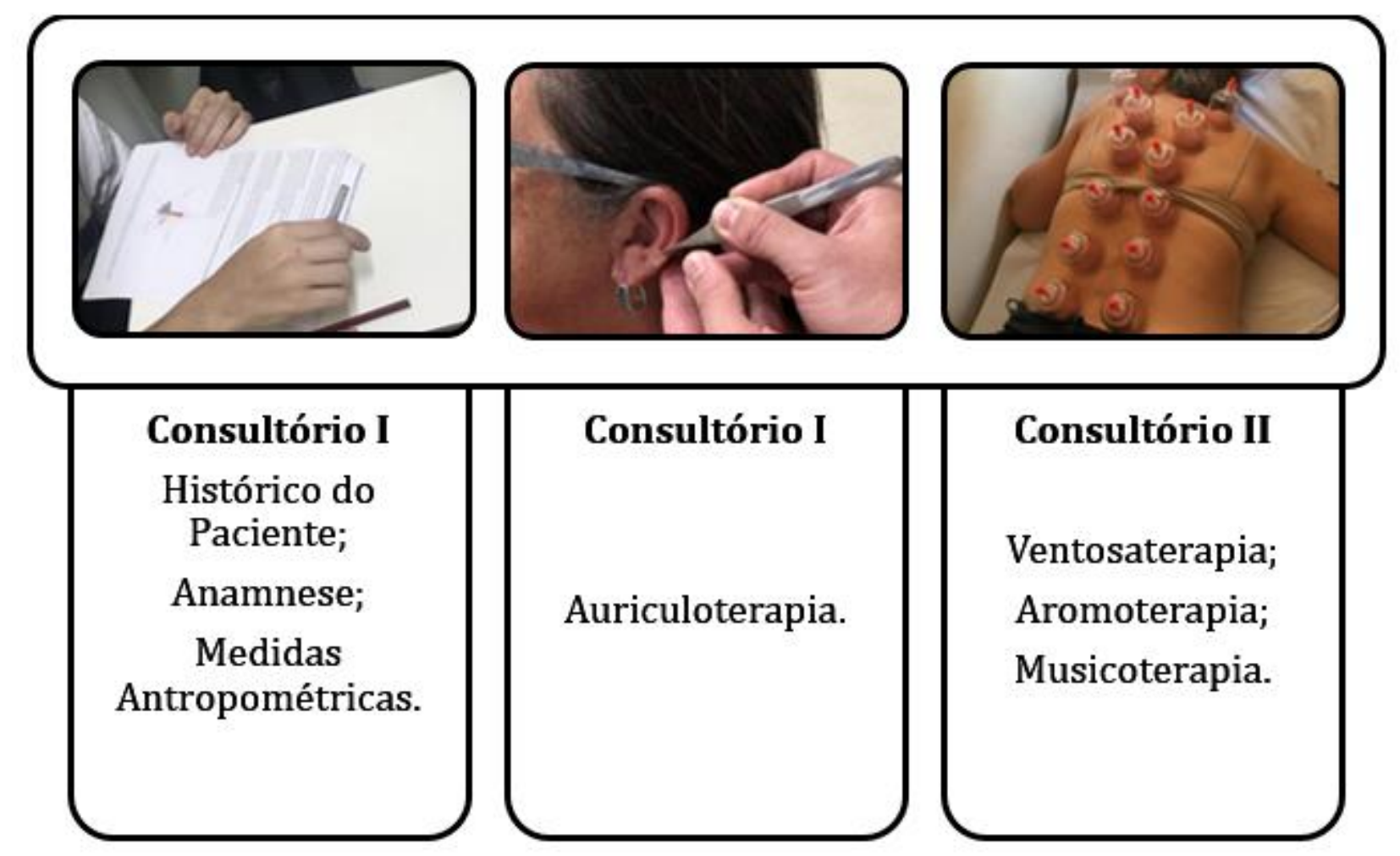

Fonte: Mundim BVR, et al., 2019.

\section{DISCUSSÃO}

A prática do cuidar foi evoluindo juntamente com a ascensão das diversas tecnologias, porém, essa evolução foi acompanhada de uma estratificação que causou um isolamento de tais práticas daquilo que seria social/coletivo. Essa objetificação do cuidado segmentou ainda mais os processos de trabalhos em saúde, isso expressava que as visões do cuidado estavam relacionadas a um reducionismo biológico. Entretanto, todo esse processo tem sido amplamente criticado, em detrimento de uma busca constante do cuidado voltado a um indivíduo complexo e holístico (SCHVEITZER MC, et al., 2012).

Assim, ressurgem práticas que buscam uma ressignificação e valorização do indivíduo como as práticas oriundas da "medicina alternativa". Nesse contexto, o Brasil implementou no SUS, em 2006, as Práticas Integrativas e Complementares (PIC's) como opções de trabalho para a abordagem primaria (SCHVEITZER MC, et al., 2012). As PIC's aplicadas no tratamento dos pacientes de lombalgia nesse estudo foram ventosaterapia, auriculoterapia, aromoterapia e musicoterapia; visando, além do tratamento propriamente dito, deixar o ambiente mais acolhedor e humanizado para os pacientes.

A ventosaterapia é uma terapia complementar da Medicina Tradicional Chinesa, indicada para redução da dor crônica, envolvendo a aplicação de copos de diferentes materiais, em uma área de dor e/ou acupontos, pelo uso de aparelhos de vácuo ou calor (MOURA CC, et al., 2018). Esse método foi aplicado em pacientes com lombalgia da UBS, pelo uso de copos associados ao vácuo. Após o tratamento semanal, os pacientes afirmaram que a dor sentida, avaliada pela EVA da dor, diminuiu. Corroborando com esses dados, uma revisão sistemática com metanálise que identificou 611 estudos, e destes 16 foram submetidos a análise qualitativa e 10 para análise quantitativa, constatou redução significativa do escore de intensidade da dor nas costas mediante uso da ventosaterapia, ressaltando a importância de estabelecer protocolos para seu uso (MOURA CC, et al., 2018). 
A auriculoterapia, outra técnica utilizada, consiste no estimulo mecânico de certos pontos específicos no pavilhão auricular, com a intenção de aliviar dores e tratar problemas psíquicos e físicos (MOURA CC, et al., 2019). O pavilhão auricular é considerado uma área importante, visto que possui um microssistema, que pode refletir todas as mudanças fisiopatológicas em membros, tronco, tecidos e dos órgãos de tecido (SOUZA MP, 2012). Dessa forma, a auriculoterapia foi utilizada como um método associado à ventosaterapia, para redução da lombalgia. Em um ensaio clínico randomizado em 110 pessoas com distúrbios musculoesqueléticos nas costas, a auriculoacupuntura apresentou-se como uma terapia alternativa para redução da intensidade da dor crônica (MOURA CC, et al., 2019).

Concomitante com a ventosaterapia e auriculoterapia, foram utilizadas, ainda, a musicoterapia que se constitui como o uso de sons e instrumentos capazes de promover evolução terapêutica dos pacientes (BRUSCIA K, 2000). Na prática aplicada, tal PIC foi utilizada com a finalidade de deixar o ambiente mais atrativo e promover um momento de relaxamento para o paciente. Juntamente com a musicoterapia, foi realizada a aromoterapia, por meio de óleos essenciais, extraídos de flores, folhas ou raízes de plantas, e outros tipos de fragrâncias com a intenção de melhorar o bem-estar físico e psicológico, complementando as outras PIC's, sendo aplicadas diretamente na pele por meio de massagens como método de relaxamento e colocado para ser difundido no ambiente, deixando a sala ainda mais agradável (TISSERAND R, 2004).

Diante do exposto, observa-se que durante as atividades exercidas na matéria de Interação Comunitária II foi possível trabalhar diversas áreas importantes para a formação médica. Dentre elas, a aplicabilidade das PIC's em tratamentos na atenção primaria; isso se comprova pela eficácia que foi observada a cada dia de atendimento no ambulatório. Assim, considera-se que as PIC's utilizam recursos terapêuticos baseados em conhecimento tradicionais e científicos voltados para a prevenção e tratamento de doenças, como a lombalgia.

A experiência da utilização da auriculoacupuntura, ventosaterapia, musicoterapia e aromoterapia, realizadas em ambulatórios de práticas integrativas e complementares e aplicadas em um grupo especifico de pessoas acometidas por lombalgias, foi possível observar resultados significativos em relação ao relaxamento muscular, tranquilidade, redução do estresse, ansiedade e da dor lombar em pelo menos dois níveis da EVA da dor de uma sessão a outra, segundo relatos dos pacientes.

\section{REFERÊNCIAS}

1. ABREU ATJB, RIBEIRO CAB. Prevalência de lombalgia em trabalhadores submetidos ao programa de Reabilitação Profissional do Instituto Nacional do Seguro Social (INSS), São Luís, MA. Acta Fisiatr., 2010; 17(4): 148-152.

2. ACHUTTI A, AZAMBUJA MIR. Doenças crônicas não-transmissíveis no Brasil: repercussões do modelo de atenção à saúde sobre a seguridade social. Ciência \& Saúde Coletiva, 2004; 9(4): 833-840.

3. BRASIL. Ministério da Saúde. Secretaria de Atenção à Saúde. Departamento de Atenção Básica. Política Nacional de Práticas Integrativas e Complementares no SUS. Brasília, DF: MS; 2006.

4. BRUSCIA K. Definindo Musicoterapia. Rio de Janeiro: Enelivros; 2000.

5. COUTINHO ATQ, et al. Comunicação social e independência funcional em idosos de comunidade coberta pela estratégia saúde da família. Revista CEFAC, 2018; 20(3): 363-373.

6. UNICESUMAR - Centro Universitário de Maringá (UNICESUMAR). Plano de ensino do curso de medicina. 2019.

7. GOMES MRA, et al. Lombalgia gestacional: prevalência e características clínicas em um grupo de gestantes. Rev Dor., 2013; 14(2): 114-7.

8. MOURA CC, et al. Ventosaterapia e dor crônica nas costas: revisão sistemática e metanálise. Revista Latino-Americana de Enfermagem., 2018; 26: e3094.

9. MOURA CC, et al. Efeitos da auriculoacupuntura na dor crônica em pessoas com distúrbios musculoesqueléticos nas costas: ensaio clínico randomizado. Revista da Escola de Enfermagem da USP., 2019; 53: e03418.

10. NAHAS MV, et al. O pentáculo do bem-estar-base conceitual para avaliação do estilo de vida de indivíduos ou grupos. Revista Brasileira de Atividade Física \& Saúde, 2000; 5(2): 48-59.

11. SANTOS TR, et al. Perfil socioeconômico-demográfico do beneficiário do Instituto Nacional do Seguro Social aposentado por invalidez e suas causas, no Estado da Paraíba, no quinquênio 2007-2011. Revista Brasileira de Estudos de População, 2012; 29(2): 349-359. 
12. SCHMIDT MI, et al. Chronic noncommunicable diseases in Brazil: burden and current challenges. Lancet., 2011; 377(9781):1949-61.

13. SCHVEITZER MC, et al. Práticas Integrativas e Complementares na Atenção Primária em Saúde: em busca da humanização do cuidado. O Mundo da Saúde., 2012; 36(3): 442-451.

14. SILVA MC, et al. Dor lombar crônica em uma população adulta no Sul do Brasil: prevalência de fatores associados. Cad Saude Publica., 2004; 20(2): 377-85.

15. SOUZA MP. Tratado de Auriculoterapia. Brasília: Novo Horizonte; 2012.

16. TESSER CD, et al. Práticas Integrativas e Complementares na Atenção Primária à Saúde brasileira. Saúde em Debate., 2018; 42(spe1), 174-188.

17. TISSERAND R. The art of aromatherapy. 2nd ed. Saffron Walden: CW Daniel; 2004.

18. WHO - World Health Organization. Preventing chronic diseases: a vital investment. Geneva: 2005.

19. WHO - World Health Organization. Global status report on noncommunicable diseases 2010. Geneva: 2011. 